\title{
CloT-Robot: Cloud and IoT Assisted Indoor Robot for Medicine Delivery
}

\author{
Huiru Cao, Xiaofeng Huang, Jianyi Zhuang, Jianqiang Xu, Zening Shao \\ Nanfang College of Sun Yat-sen University, School of Electrical and Computer Engineering, \\ Guangdong, Guangzhou, China \\ *xiaocao0924@163.com
}

\section{Keywords: Internet of Things; Medicine delivery; Mobile robot; Cloud technology}

\begin{abstract}
At present, there is increasing requirements for updating the healthcare system by introducing the new information and communication technologies, especially for indoor domains. However, the current system and healthcare robot are restricted to remote monitoring and medicine delivery research, cannot meet the actual demand for the aged. We presented a novel system framework and designed a CIoT robot (Cloud and IoT Assisted Indoor Robot), which based on cloud technology and Internet of Things (IoTs). The medicine robot delivery system based on multi-core embedded system, RFID, IEEE802.11 communication protocol, and cloud technology. Finally, the experiments and measured the performances of the system are constructed. The experimental results show that the well performance and feasibility of the system.
\end{abstract}

\section{Introduction}

As known, the important part of good medical care system is the indoor healthcare system. It became a focus research in western developed countries with the continuously change of world's population structure in recent years [1]. In China, the part of over 60 years old will be the fastest increase and it accounts for $7.63 \%$ to $10.35 \%$ of the total population since 1982 , which became the elderly population structure. Moreover, it will reach 15.23\% in 2020 and 24.28\% in 2040 [2, 3], which called "The ageing peak stage". Meanwhile, more and more oldster with some common disease like hypertension, diabetes, cerebral thrombosis, and sudden illness like cardiopathy needs to be taken care promptly. With the development of people's living standard and the information technology, provide for the elderly in society or at home is the key to solve the problem of social provide for the elderly in China. However, many problems have been erupted like lack of funds, talents and the Hospital Information System, so that current medical system is hard to fit the actual and development. Therefore, it is necessary to invent new equipment about indoor medical care system.

People from all over the world have done amount of research on it. For example, Marco [4] led his team to make up an indoor health care system which based on embedded system to protect someone who injured in the house, and the results proved the feasibility of that method. Chong [5] and his team designed a home pharmaceutical monitoring system which based on household robot and wireless sensor network, and the results of experiment proved that the household robot may improve the ability of wireless sensor and family monitoring system. In [6], the authors developed a new computational algorithm for accurate patient emotional state classification in interaction with nursing robots during medical service. Generally speaking, the present research about indoor health care system or other equipment is tending to monitor vital signs. It has lots of shortcomings like the low information and lack of remote monitoring, so we designed a robot who can deliver medicine to cover the above shortage. The robot is based on cloud and IoTs technology. It has remote mobile terminal and PC terminal management system. Last but not least, we tested the system and validated the rationality and feasibility on it.

The remainder of this paper is organized as follows. Framework of healthcare system based on IoT and cloud is briefly introduced in Section II. In section III, hardware design of the system for CIoT robot is given. Section IV, the CIoT robot Software design and Medicine delivery algorithm is 
analyzed. Experiment and implementation is done in section V. Finally, Section VI presents the overall conclusions.

\section{Framework of Healthcare System Based on Cloud and IoT}

The framework of indoor health care system based on could and internet of things technology may improve the system' intellectualization and informatization. We can see the layers of health care system from Figure 1(a). It includes sensing layer, network layer technology, and application layer technology. In the framework, sensing layer aware system is divided into data collection and information processing; the network layer realizes information interaction and data communication; application management is made up of message open authoring system, IoT application and support system make up the applied layer.

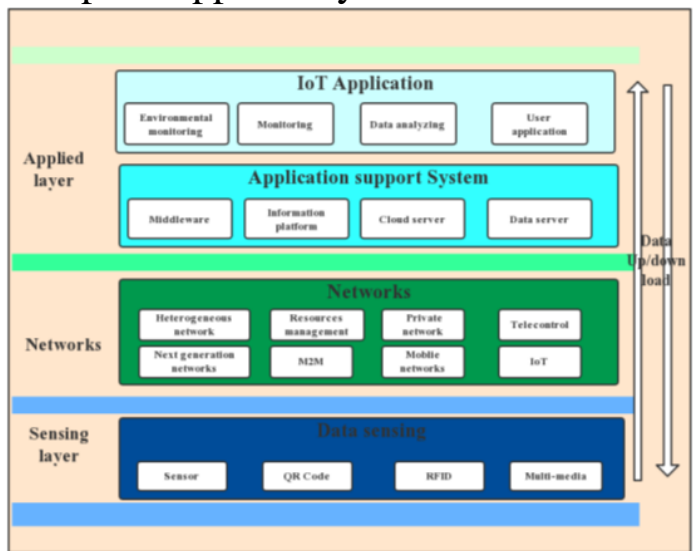

(a) The IoTs Technology Framework

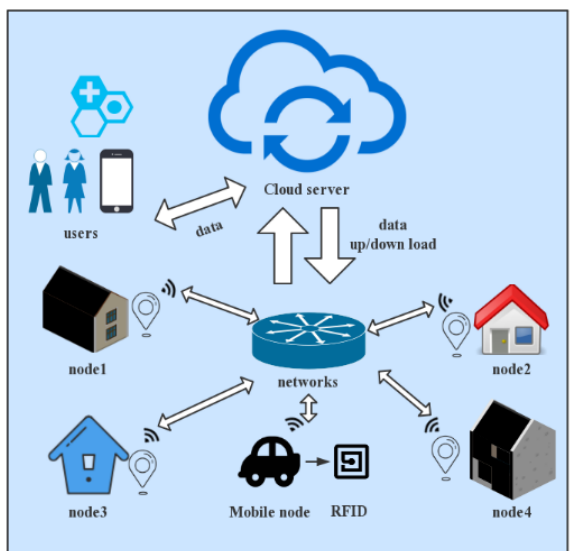

(b)The IoTs Functional Architecture

Figure 1. Technology framework and Functional architecture diagram of internet of things

The robot system of medicine delivery is divided into six parts: network node control, the display of on-line system position, medicine distribution based on RFID (Radio Frequency Identification Devices), data gathering from indoor environment, cloud storage and the client. We can see conceptual diagram from Figure 1(b). Network node control: control the sort and amount of the medicine and the condition in every room through a terminal. The display of on-line system position: show the robot position and display on the terminals when it begins to work. Medicine distribution: decide the condition about medicine in every room through the sensors. Data gathering from indoor environment: display the environmental conditions in real time on terminals. Cloud storage: store and monitor the environment of pharmaceutical monitoring home. Client: show conditions of the system more intuitionistic and visible.

\section{Hardware Design for CIoT Robot}

3.1 General hardware design. The hardware system consists of indoor perception nodes, a robot deliver medicine, and sensor network nodes.

Table I. Hardware Module

\begin{tabular}{lll}
\hline Module & Interface types & Function \\
\hline MFRC-522 RFID module & SPI & Read and modify the information of node \\
Fudan card70 (M1card) & ISO14443 & As the control of moving node \\
infrared module & AO & Trailing \\
PM2.5sensor & AO & Test the point of PM2.5 index \\
SG90 Steering engine & Digital & The action for dispensing medicine \\
camera & CSI & Cloud monitoring \\
ESP8266WIFI module & USART & Data transmission \\
\hline
\end{tabular}


We can learn the hardware module consist of multi-core ARM by master control, STM32F103RC processor by assisting control, MFRC522 module, all kinds of sensors, cameras and so on from table 1. The module connects with digital interface, SPI (Serial Peripheral Interface), AO (Analog Output), CSI (COMS Sensor Interface), USART (Universal Synchronous/Asynchronous Receiver /Transmitter) to accomplish the function of perception of the system in need, data delivery, medicine delivery, etc. Figure 2 (a) shows the overall of the robot.

3.2 Hardware design for robot. In Figure 2(b), the system uses a high performance of STM32F103RC microprocessor which based on ARM Cortex-M3 kernel, which has the topic advantages is high performance, low cost, low power consumption. When executing code through flash, lower power to $36 \mathrm{~mA}$ as about $0.5 \mathrm{~mA} / \mathrm{MHz}$ practically with the built-in SRAM of $96 \mathrm{~K}$ when frequency is $72 \mathrm{MHz}$ of the clock. Besides, it also uses the high performance Multi-core embedded system like the Raspberry Pi of the 3rd generation of 64 bit ARMV8 processor with $1.2 \mathrm{GHz}$ four cores Broadcom CEM2837 and BCM43143WIFI module onboard and so on to ensure the function of medicine delivery more stably. In addition, the system uses RC522 module to operate the read-write card. As a member of serious of high integration read-write card chip with $13.56 \mathrm{MHz}$ non-contact communication, RC522 is a contactless read-write card chip with low voltage, low cost, small size according to the "three expressions" application in NXP company. It also has many excellent functions such as integrated modulation and demodulation circuit, the support of ISO/IEC 14443 Type A and MIFARE\&reg, communication protocol, SPI interface of 10Mbit/s, flexible break mode, which is propitious to get the information from card fleetly and steadily.

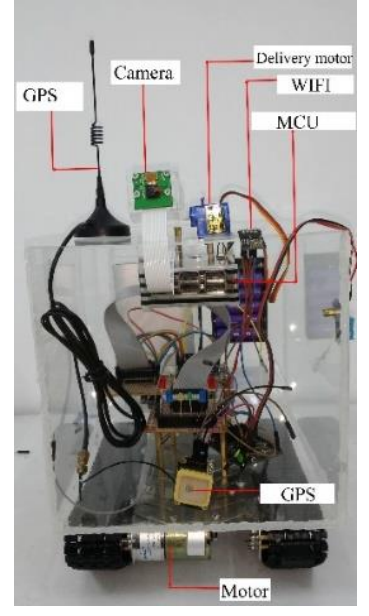

(a)Robot Architecture

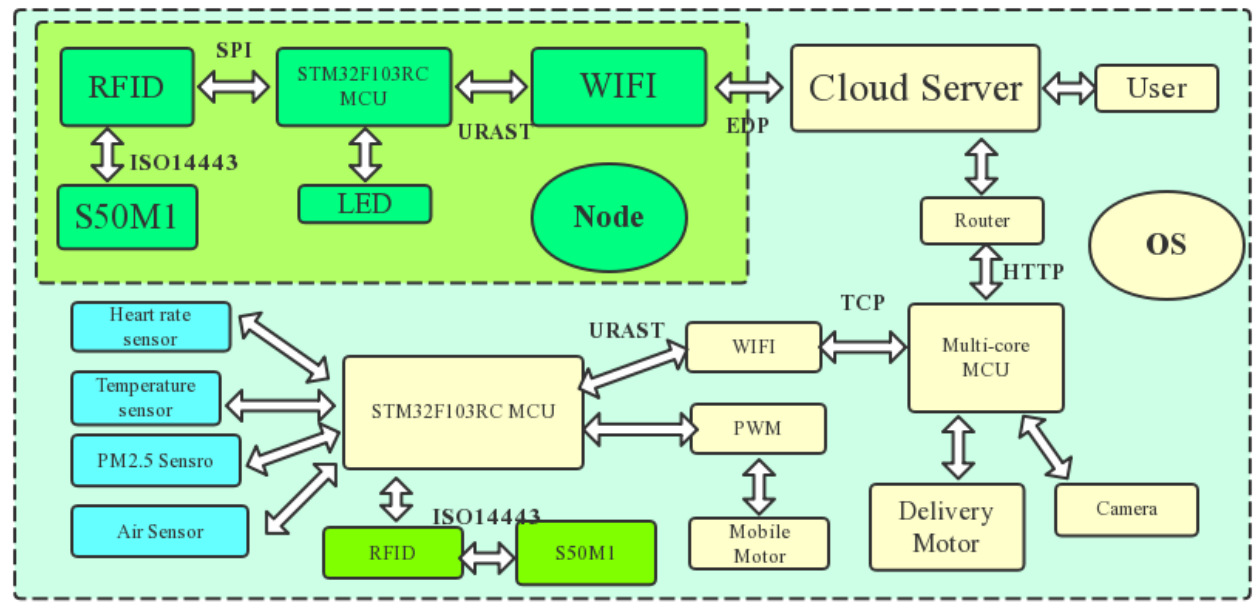

(b) Hardware Design Block Diagram

Figure 2. The Robot architecture and Hardware design block diagram

When the system staring, the read node information is stored in string variable by STM32F103RC and the string variables are sent to the multicore embedded data processing center by ESP8266WIFI module of USART serial driver. After the multicore embedded receiving the data, it will determine the type of data, and it will control the move of steering engine to complete the action of delivering medicine by the time base pulse of the pin output at that time or monitor the circumstances in the room by using CSI cable camera. When the STM32F103RC processor star, the system will configure WIFI module to connect the hotspot with net by USART input instructions and configure RC522 module by SPI serial ports input instructions. Then WIFI module will download the data on cloud server by EDP protocol, and deliver the information. If the system detects the re-back data has no control command, it will not open the antenna of RC522 module and not do the read-write operation. On the contrary, it will open the antenna of RC522 module and write card indicator light and read its new data to the node by ISO14443A.

\section{CIoT robot software design and medicine delivery algorithm}

4.1 Design function for robot. When the system starts to run, multi-core embedded plays the part of client and STM32F103RC acts as a server, which connects the same hotspot. Then the multi-core 
embedded offers multithreading to control the data from ATM32F103RC by WIFI module which is used to monitor actions (Such as the read-write condition of RC522 and data of sensor, etc.). After receiving the data of WIFI module, it will analyze the data types at the first time. For example, RC522 read the data of node, then judge whether to open the camera and to deliver medicine or not. If all goes well, the data of indoor environment sensor will be uploaded promptly to cloud, else return a mode of waiting to accept the data.

When the system starts, seeking mark function of STM32F103RC controlling motor will starts up. Drive sensors collect the indoor environment data incessantly, and send the data to the center of data handle multi-core embedded every 4 seconds. STM32F103RC will judge if it has readable data's node, if the answer is yes, it will store the data into string variables, and send to the center of data handle by WIFI module, then re-back to the movement of tracing or re-back the movement of tracing firsthand.

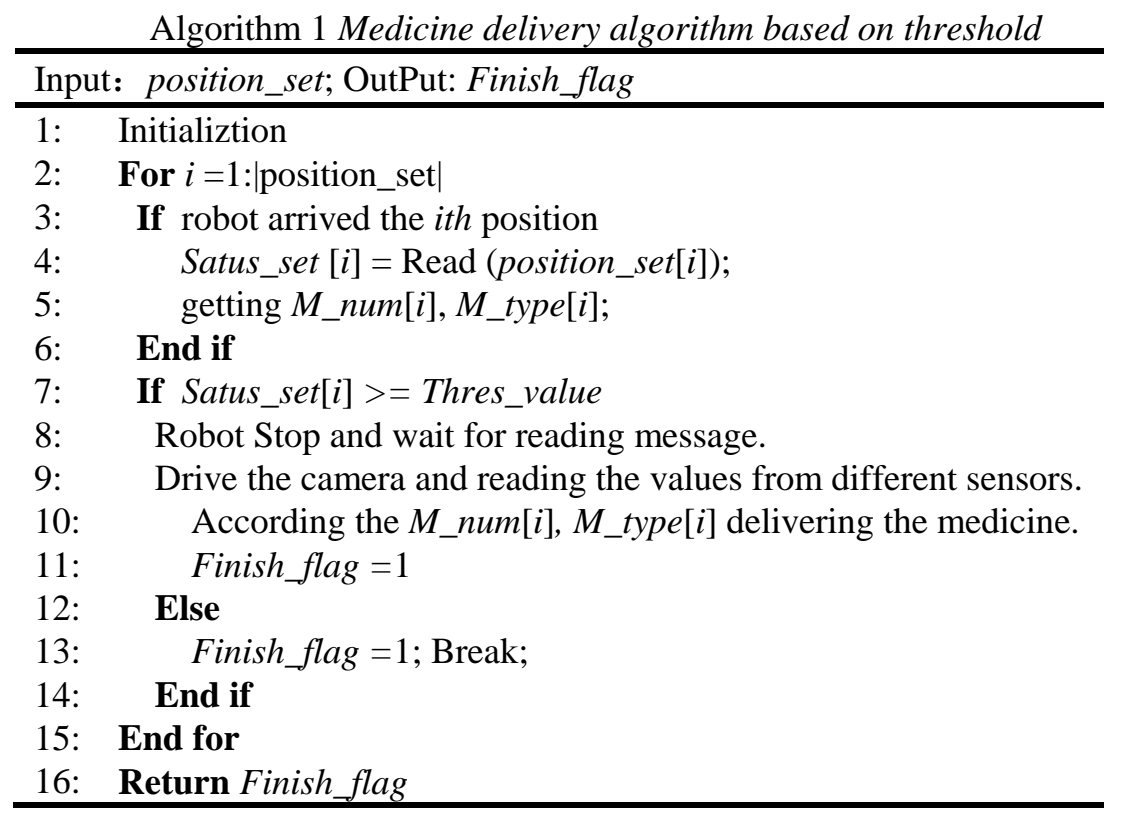

4.2 Medicine delivery threshold algorithm. According to the characteristic of system design, we propose the medicine delivery algorithm which based on threshold. The specific algorithm design is shown in Algorithm 1. This algorithm divided into three parts, first is the position set of medicine delivery information. When the robot moves to the ist position, the system will read the state information of Satus_set $[i]$ from IC card, and get the kind and amount of medicine like $M \_n u m[i]$, $M_{-}$type $[i]$. Secondly, it will judge the thresh value. If the value above or equal threshold, the moving robot will stop the present position to finish reading information and collecting image and delivering medicine. Then assign that finish_flag being 1, otherwise finish the delivery and let finish_flag be 0 . Finally, algorithm returns finish_flag.

\section{Experiments and Implementation}

In the lab, we establish an experimental environment to test the time of data uploading to cloud computing service, the function of prescription and node writing. System always uploads data and stores in cloud, at the same time; it will analyze the function according to the time and details of data uploading. The least time it costs is $2.1 \mathrm{~s}$ and the longest time is $7.5 \mathrm{~s}$, so we can know that the average time it costs is $5.21 \mathrm{~s}$, and the error of uploading time is less than $1 \mathrm{~s}$, when every data uploaded to the cloud. In a word, our system's data uploading and the storage of cloud are very smoothly.

According to the node information, our system can deliver different medicines. We check and write down the steering machine how many times it costs and how many electric current it uses when delivering medicines. The maximum current reach $1.923 \mathrm{~A}$ and the minimum current is $0.131 \mathrm{~A}$, 
which the average current is $0.77 \mathrm{~A}$. The count of medicines depends on the time of a machine costs. The more counts, the more time it costs, when the average time of delivering per medicine is $4.19 \mathrm{~s}$.

Each special node is allocated the unique network nodes to acquire the messages from the online and write them down original card M1 as well as make the system respond a relevant action. Meanwhile, we randomly extracted five experiments and recorded the time that network nodes will be successfully written in the card in the test. The longest time that nodes were successfully written in the card is 50 seconds and the average time is 48.3 seconds.

\section{Conclusions}

At present, the period of a rapid aging population is coming, especially in developing countries. The healthcare system needs to the new paradigm and medical robots. However, the present ones cannot meet these demands. After a survey of the present iatrical system at home and abroad, an intelligent smart frame and CIoT robot are presented on account of the cloud and IoT technology. As a matter of fact, we build a new indoor CIoT robot for delivery medicine through the multi-core embedded system, the communication protocol of RFID and IEEE802.11, open cloud platforms. We also design a management system of remotely mobile terminal and PC terminal. Finally, for verifying rationality and feasibility of the proposal, we build a relevant experimental environment and analyze the performances. In the future, we will further optimize the CIoT Robots and build a large-scale experiment for the proposal.

\section{Acknowledgment}

This work is partly supported by the Water Resource Science and Technology Innovation Program of the Guangdong Province, China (No. 2016-18) and Guangdong 2017 college students' training program for innovation and Entrepreneurship (No. 201712619011).

\section{References}

[1] Torkestani S S, Julien-Vergonjanne A, Cances J P. Indoor optical wireless system dedicated to healthcare application in hospital[C]// International Symposium on Communication Systems Networks and Digital Signal Processing. IEEE, 2010:542-546.

[2] Wang Z, Sun T, Li G. Regional Differences and Evolutions of Population Aging in China[J]. Population Research, 2013:37-42.

[3] Zhou Q L A, Min-jie b H U. A Solution to the Problem of Remote Medical Insurance for Chinese Senior Citizens[J]. Journal of Jiangxi University of Finance \& Economics, 2009.

[4] Mercuri M, Garripoli C, Karsmakers P, et al. Healthcare System for Non-invasive Fall Detection in Indoor Environment [J]. Applications in Electronics Pervading Industry, Environment and Society, 2016, 351:145-152.

[5] Yu C, Chen X. Home monitoring system based on indoor service robot and wireless sensor network [J]. Computers \& Electrical Engineering, 2013, 39(4):1276-1287.

[6] Swangnetr M, Kaber D B. Emotional State Classification in Patient-Robot Interaction Using Wavelet Analysis and Statistics-Based Feature Selection[J]. IEEE Transactions on Human-Machine Systems, 2012, 43(1):63-75. 